\title{
Evolución de los precios y cotización de las divisas
}

\author{
Joaquin Arriola Palomares
}

Decir que el colón está "sobrevaluado" es algo tan común en estos días, que nadie se ha puesto a reflexionar sobre qué significa esta expresión, tanto técnica como políticamente. En este trabajo pretendemos mostrar que los argumentos que se utilizan para tal afirmación son no solo débiles sino políticamente orientados al reforzamiento de los sectores hegemónicos de la clase dominante salvadorena.

El argumento que se esgrime en defensa de la hipótesis de la sobrevaluación es el siguiente: considerando la inflación interna como factor de competividad exterior, se afirma la existencia de un tipo de cambio real" que sería el tipo de cambio nominal más el diferencial de inflación del país con respecto a los principales socios comerciales (BCR) o respecto a un "índice de precios internacionales" (Fusades). Si el indice de precios local muestra una tendencia al alza mayor que la media ponderada de los índices de precios de los socios comerciales, entonces, el tipo de cambio real seria mayor que el nominal, postulándose la necesidad de la devaluación.

Esta definición, no por muy difundida presenta menos inconsistencias. En primer lugar vincular el manejo del tipo de cambio con la evolución de la balanza comercial no es correcto, puesto que es la balanza de pagos total y no solo la balanza por cuenta corriente la que define la situación externa de una economia, y por tanto la que se refleja en la evolución del precio relativo de la moneda nacional.

Además, están los problemas técnicos existentes para estimar cuan- 
titativamente la importancia de cada socio comercial y para la identificación de dichos socios. Las mercancias relevantes para la estimación de un índice de competitividad de precios o de costes, son:

a) las mercanclas de exportación de los demás paises sustitutivos de las exportaciones del propio pais

b) las mercancias producidas para el mercado nacional en los paises al cual se dirigen nuestras exportaciones, sustitutivas de estas

c) las mercancias de origen extranjero susceptibles de sustituir la producción nacional.

Por lo tanto, a través del comercio internacional, no es solo la producción para la exportación la que tiene que competir con las exportaciones de los otros paises; también la producción para el mercado interno puede sufrir pérdidas de competitividad si los costes de producción aumentan más rápido que en otros paises.

En tercer lugar, si en relación con la producción para el mercado interno, el indice general de precios es el indice relevante, no ocurre lo mismo con la producción para la exportación. Los precios relevantes para medir la competitividad externa no es en todo caso el IPC, sino los precios de exportación, confrontados con los precios de exportación de nuestros competidores.

Cuanto mayor indice de concentración muestra la estructura de exportaciones de un pais, menos relevante es el IPC para medir la competitividad vía precios. A la inversa, el IPC se aproxima a un indicador adecuado de dicha competitividad cuando el grado de concentración de exportaciones es mínimo. Si en el caso de los Estados Unidos, por poner un ejemplo, el indice general de precios puede ser una aproximación a la competitividad de precios tanto internos como extemos. En el caso de El Salvador, evidentemente, como en el de los demás paises centroamericanos, la evolución del indice general de precios o inflación no es el más adecuado, y si una selección de unos pocos precios de exportatión.

En el caso de El Salvador, atentiendo a la estructura de sus exportaciones, podemos descomponer los precios de las exportaciones de la siguiente manera:

$$
P=P^{\prime}+a P^{a}+v P^{v}+o P^{\circ}
$$

donde $\mathrm{P}^{\prime}$ es el precio del café, $\mathrm{P}^{\mathbf{a}}$ es el precio del azúcar, $\mathrm{P}^{v}$ es el precio de las materias textiles, sus manufacturas y calzado (vestuario) y $P^{\circ}$ es el precio de las demás exportaciones; $f, a, v, 0$ son los porcentajes que 
representan en el conjunto de las exportaciones los rubros senalados. Tomando para las ponderaciones la composición de las exportaciones en cifras absolutas para los anos 1988-1990, tenemos que:

$$
\begin{aligned}
& f=52,1 \\
& a=2,3 \\
& v=12,4 \text { y, por diferencia } \\
& 0=33,2
\end{aligned}
$$

es decir, la evolución de los precios del café, azúcar y vestuario, explicarian dos tercios de la competitividad relativa de precios de las exportaciones salvadorenas.

Si nos fijamos en la evolución de los precios de dichos rubros, tenemos que si en 1991 la variación anual al mes de septiembre respecto a igual fecha del ano anterior del IPC fue del 16,11. Para los precios de exportación considerados, tenemos por su parte la siguiente evolución:

$$
\begin{aligned}
& P^{\prime}=-10,5 \\
& P^{2}=9,8 \\
& P^{v}=\quad 5,9 \\
& P^{0}=16,1 \text { (en este caso, tomamos como indice el IPC) }
\end{aligned}
$$

que tomando en consideración nuestra ponderación del peso de cada rubro, nos da una evolución de los precios de las exportaciones de

$$
P=(0.5 \times-10,5)+(0.02 \times 9.8)+(0,1 \times 5,9)+(0,3 \times 16,1)=8,2
$$

Es decir, la inflación implícita en los precios de exportación es aproximadamente la mitad de la reflejada en el IPC.

Aun dando por bueno el adsurdo teórico, defendido entre otros por Fusades (Boletín Económico y Social num. 70) de la existencia de un "índice de precios mundial", tan posible es que este crece un $5 \%$ por ano como que crece un $8 \%$, con lo cual el tipo de cambio no estaría en absoluto "sobrevaluado".

Siendo más rigurosos, para comparar el efecto sobre la competitividad de los diferenciales en la evolución de los precios, habria que conocer la evolución de los precios de exportacion de los nubros más importantes en los posibles competidores extranjeros.

Aqui conviene hacer otra consideración particular. El precio de los coloniales es el mismo para todos, con lo cual no se ve afectado por la inflación interna, sino por la evolución de los costes de producción en 
los diferentes paises exportadores. El control de la comercialización en los mercados exteriores por las companias multinacionales, hace que los precios internacionales de los productos coloniales -los únicos relevantes pues en ellos se traduce la mayor parte de la realización del valor de este tipo de mercancias - sean precios de monopsonio (monopolio de demanda). La imposibilidad de incrementar en contenido en trabajo (medio) de los productos coloniales -es decir, sus escasas posibilidades de incrementar el valor agregado en la fase de producción primaria hace que, ante una evolución negativa de los precios internacionales, la única posibilidad de incrementar las ganancias de los productores capitalistas es aumentar la producción (superficie cultivada) 0 reducir el coste de la mano de obra. Los precios no se pueden reducir por medio de una devaluación, que por el contrario aumentará los precios de los insumos importados, y por tanto los costes de producción. Pero siempre menos que la ganancia extra de los exportadores en moneda nacional:

$$
\Delta C<\Delta B^{x} t
$$

De esta forma, las ganancias en moneda nacional de los exportadores de productos coloniales aumentarán con la devaluación, sin que esto signifique ninguna variación en su competitividad externa.

Siendo C: costes de producción (en moneda nacional), B: precio de venta (en dólares); t: tipo de cambio (unidades de moneda nacional por cada unidad divisa/dólar). Según las estimaciones del Ministerio de Trabajo, los costes de producción del café oro en las superficies con un rendimiento de 20 quintales por manzana es de 285 colones por quintal, para la cosecha de 1990/91. Para un tipo de cambio con el dólar de $t=$ 8 y un precio en los mercados internacionales de unos 80 US $\$$ el quintal esto significa un margen del precio de venta sobre el precio de producción del $125 \%$ (impuestos y fletes excluidos). Una devaluación que elevase $t$ hasta $t=10$, elevaria el margen hasta un $180 \%$, sin que esto significase ningún incremento en la cuota de mercado externo, o mejora en los costes de producción o en los rendimientos por manzana.

Los productos no coloniales, cuyo precio de venta internacional no está determinado por las posiciones de monopolio u oligopolio de las multinacionales, son los únicos para los cuales tiene alguna relación con la realidad la comparación de la evolución de los precios en diferentes paises en relación con la competitividad aunque tampoco en este caso tiene el sentido que le quieren dar los economistas oficiales, paraoficiales y quintacolumnistas. 
En primer lugar, habria que relacionar estos precios con los de los competidores en los mercados a los cuales se dirigen nuestras exportaciones, y con los precios domésticos para estos mismos paises, de forma que la relación relevante es

$$
P^{x} /\left(p_{i}^{x}\right) t
$$

donde $\mathrm{P}^{\mathbf{x}}=$ variación del precio ponderado de las exportaciones nacionales no coloniales; $t=$ tipo de cambio, $i=$ los paises competidores en los mercados de destino y los propios paises de destino de nuestras exportaciones no coloniales, y $\mathrm{P}^{\mathrm{x}}$ es la suma ponderada de las variaciones de los precios en esos paises de los productos de exportación no coloniales de nuestro pais.

Solo cuando esta relación es positiva, es decir, solo cuando la variación positiva del precio de las exportaciones no coloniales es mayor que la variación en el mismo sentido de los precios de las exportaciones extranjeras sustitutivas de las primeras, se puede hablar de un deterioro de competitividad por la via de la variación de los precios relativos, y por tanto postularse la devaluación como medida de compensación coyuntural.

Pero este manejo del tipo de cambio no garantiza en absoluto un alivio de la balanza comercial, que depende en todo caso del cumplimiento del teorema de las elasticidades críticas, que A. P. Lerner definia en 1944 en términos que recuerdan al análisis que aqui desarrollamos: "la condición suficiente para que una balanza comercial equilibrada se vuelva positiva tras una depreciación de la moneda nacional (efecto normal) es que siendo infinitas las elasticidades-precio de las ofertas (doméstica de exportaciones y extranjera de importaciones), la suma de las elasticidades-precio de las demandas (doméstica de importaciones y extranjera de exportaciones) sea superior a la unidad. En el caso contrario, podemos esperar que la depreciación de la moneda nacional vuelva negativa la balanza comercial (efecto perverso)". La relativa rigidez de la elasticidad-precio de la demanda de importaciones nacional, unido a la ausencia de productos de exportación para los cuales poseamos una parte predominante de las disponibilidades mundiales, permite aseverar que el efecto comercial de la devaluación se aproxima más al efecto perverso que al normal.

Además, no se puede olvidar que la devaluación genera inflación. La evolución de los precios internos -medidos por el IPC - se descompone en precios domésticos y precios importados, en la siguiente relación: 


$$
P=\propto P^{a}+\beta P m
$$

donde

$$
\alpha=([\mathrm{PIB}-\mathrm{X}-\mathrm{M}] / \mathrm{PIB}) \times 100
$$

o lo que es igual,

$$
\alpha=([C+I]+[X-M] / P I B) \times 100
$$

es decir, $\propto$ es el coeficiente de gasto doméstico sobre la producción total, $y$

$$
B=M / P I B \times 100
$$

es el coeficiente de importación.

En el caso de El Salvador, con los datos correspondientes a 1990, el valor de estos coeficientes es:

$$
\begin{aligned}
& \alpha=57,2 \% \\
& B=26,8 \%
\end{aligned}
$$

Por lo tanto, suponiendo una elasticidad precio de la demanda de importaciones igual a cero, cada punto porcentual de devaluación representa un incremento de aproximadamente 0,25 punto en el IPC, como efecto directo, además del efecto indirecto que representa el incremento de los costes de los insumos importados en los bienes de producción nacional.

La devaluación deteriora por último la relación real de intercambio. Con la devaluación, cada unidad de trabajo importado deberá pagarse con una mayor cantidad mayor de trabajo nacional (o de producto, o de valor agregado, que son formas alternativas de medir el mismo efecto). Nos encontramos con un efecto de retroalimentación entre inflación (P), competitividad $(\mathrm{C})$ y devaluación ( $\mathrm{t}$ ); una vez que se comienza utilizando la devaluación para mejorar la competitividad de las exportaciones, su efecto sobre la inflación obliga a continuar permanentemente con su utilización, generándose una espiral entre devaluaciones cada vez más profundas y alzas de los precios mayores: 


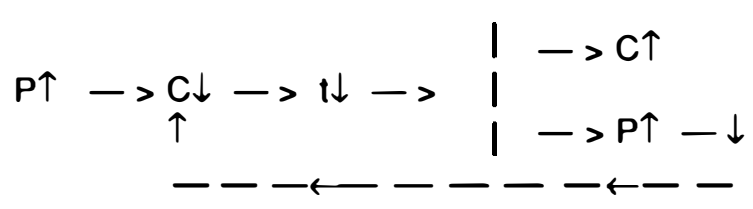

Esto está reflejado en la evolución reciente de los precios al por mayor:

$$
\text { Enero } 1978=100
$$

(3)

\begin{tabular}{|l|c|c|c|c|}
\hline Enero 89 & 350,26 & 195,99 & 272,61 & 283,15 \\
\hline Enero 90 & 400,38 & 160,43 & 321,63 & 334,50 \\
\hline Dic. 90 & 495,51 & 182,92 & 378,61 & 401,04 \\
\hline
\end{tabular}

(1): Producidos y consumidos en el pais; (2) $x ;(3): x$ - café; (4): M

Fuente: BCR, Revista Trimestral, julio-diciembre 1990

Entre enero de 1989 y diciembre de 1990, los precios de las importaciones crecen lo mismo que los de la producción nacional para el mercado interno, un $41,5 \%$, mientras que los precios de exportación incluso disminuyen un $6,5 \%$, o crecen un $39 \%$ si excluimos el café, dato este último menos relevante, por la importancia de este producto en las exportaciones totales.

La evolución de los precios para el mercado interno es la misma tanto si son productos de importación como productos nacionales. Se podria interpretar que los precios de importación varian con la inflación interna, pero no es el caso, al tratarse de indices de precios al por mayor: parece más factible la hipótesis de que los precios de importación determinan en gran medida la evolución de los costes de producción para el mercado interno, por la ausencia de producción interna de sustitución de importaciones.

En definitiva, si la devaluación parece tener mayor impacto en la configuración del mercado interno, contribuyendo en gran medida al incremento de los precios, y si el incremento de los precios se convierte en el argumento principal para justificar el deterioro de la competitividad externa de las exportaciones, al menos se debería descartar por ilógica la propuesta de devaluar para contrarrestar esta pérdida de competitividad. Solo la de consideración que muestran los economistas neolibe- 
rales y los subordinados a esta escuela, hacia el mercado interno, embarcado en su mayoria en la mistica del mercado intemacional (inexistente) y la estrategia de promoción de exportaciones (que no es una estrategia de desarrollo), permiten entender porqué se cometen errores teóricos con argumentos "técnicos" (para la realidad de El Salvador y la región centroamericana) como el "índice del tipo de cambio efectivo real", o se defiende "técnicamente" la devaluación como medida de politica económica orientada a la defensa de la competitividad de las exportaciones. Los intereses que se ven favorecidos con esta linea argumental son precisamente los de los sectores sociales y productivos más retardatarios del pais, los de quienes en más de un siglo de hegemonia han sido incapaces de modificar la normas de consumo, el patrón de especialización internacional y el modelo de acumulación vigente en EI Salvador. Curiosamente, el gobierno muestra más pragmatismo y conocimiento de la economia salvadoreña que muchos economistas que se postulan como más "modernos", "progresistas" o "altemativos": permitiendo que sea el mercado quien fije el tipo de cambio, para manejar los desequilibrios de la balanza por cuenta corriente se reducen las tasas de interés (faltaria añadir una modificación estructural en la política fiscal), se mantienen los incentivos a la exportación y se promueve una zona de libre comercio en Centroamerica (acuerdo de libre comercio con Honduras) que amplia el espacio del mercado "local" para la producción nacional. Favorecer de forma general el incremento en el corto plazo de la producción es sin duda más efectivo para estabilizar los precios internos que aumentar las ganancias de los exportadores desestabilizando el precio de las divisas. 